\title{
Characterizations of New Modified Weibull Distribution
}

\author{
G. G. Hamedani \\ Marquette University
}

\begin{abstract}
Several characterizations of a New Modified Weibull distribution, introduced by Doostmoradi et al. (2014), are presented. These characterizations are based on: $(i)$ truncated moment of a function of the random variable; (ii) the hazard function; (iii) a single function of the random variable; $(i v)$ truncated moment of certain function of the 1 st order statistic.
\end{abstract}

Keywords. Characterization; hazard function; modified Weibull distribution; truncated moment.

MSC 2010: 62E10.

\section{Introduction}

Characterizations of distributions are important to many researchers in the applied fields. An investigator will be vitally interested to know if their model fits the requirements of a particular distribution. To this end, one will depend on the characterizations of this distribution which provide conditions under which the underlying distribution is indeed that particular distribution. Various characterizations of distributions have been established in many different directions in the literature. In this short note, several characterizations of a New Modified Weibull (NMW) distribution, introduced by Doostmoradi et al. (2014), are presented in four directions. These characterizations are based on: $(i)$ truncated moment of a function of the random variable; (ii) the hazard function; (iii) a single function of the random variable; $(i v)$ truncated moment of certain function of the 1st order statistic. Our characterizations of NMW distribution $(i)$ will employ a version of an interesting result due 
to Glänzel (1987) (Theorem 1 of Section 2 below). The advantage of these type of characterizations is that, cumulative distribution function, $F$, need not have a closed form and are given in terms of an integral whose integrand depends on the solution of a first order differential equation, which can serve as a bridge between probability and differential equation.

The $c d f$ (cumulative distribution function) $F(x)$ and $p d f$ (probability density function) $f(x)$ of NMW are given by

$$
F(x)=F(x ; \alpha, \beta, \gamma, \lambda)=1-\exp \left\{-e^{\alpha x^{\gamma}}+e^{-\beta x^{\lambda}}\right\}, \quad x \geqslant 0,
$$

and

$$
\begin{aligned}
f(x) & =f(x ; \alpha, \beta, \gamma, \lambda) \\
& =\left(\alpha \gamma x^{\gamma-1} e^{\alpha x^{\gamma}}+\lambda \beta x^{\lambda-1} e^{-\beta x^{\lambda}}\right) \exp \left\{-e^{\alpha x^{\gamma}}+e^{-\beta x^{\lambda}}\right\}, \quad x>0,
\end{aligned}
$$

where $\alpha>0, \beta>0, \gamma \geqslant 0$ and $\lambda \geqslant 0$ are parameters. For further properties and the domain of applicability of NMW distribution, we refer the interested reader to Doostmoradi et al. (2014).

The presentation of the content of this work is as follows: In Section 2, we present our characterization results based on truncated moment of a function of the random variable. Section 3 is devoted to characterization of NMW in terms of the hazard function. Section 4 deals with characterization of NMW in terms of the conditional expectation of a function of the random variable. Finally, in Section 5, we present a characterization of NMW in terms of the 1 st order statistic.

\section{Characterizations Based on Truncated Moment}

In this section we present characterizations of NMW distribution in terms of truncated moment. As mentioned in the Introduction, our characterization results presented here will employ a version of an interesting result due to Glänzel (1987) (Theorem 1, below). The advantage of the characterizations given here is that, $c d f F$ need not have a closed form and are given in terms of an integral whose integrand depends on the solution of a first order differential equation, which can serve as a bridge between probability and differential equation. 
Theorem 1. Let $(\Omega, \mathcal{F}, \mathbf{P})$ be a given probability space and let $H=[a, b]$ be an interval for some $a<b(a=-\infty, b=\infty$ might as well be allowed $)$. Let $X: \Omega \rightarrow H$ be a continuous random variable with the distribution function $F$ and let $g$ be a real function defined on $H$ such that

$$
\mathbf{E}\{g(X) \mid X \geqslant x\}=\eta(x), \quad x \in H,
$$

is defined with some real function $\eta$. Assume that $g \in C^{1}(H), \eta \in C^{2}(H)$ and $F$ is twice continuously differentiable and strictly monotone function on the set $H$. Finally, assume that the equation $\eta=g$ has no real solution in the interior of $H$. Then $F$ is uniquely determined by

$$
F(x)=\int_{a}^{x} C\left|\frac{\eta^{\prime}(u)}{\eta(u)-g(u)}\right| \exp \{-s(u)\} d u,
$$

where the function $s$ is a solution of the differential equation $s^{\prime}=\frac{\eta^{\prime}}{\eta-g}$ and $C$ is a constant, chosen to make $\int_{H} d F=1$.

Proposition 1. Let $X: \Omega \rightarrow(0, \infty)$ be a continuous random variable and let $g(x)=e^{-e^{\alpha x^{\gamma}}+e^{-\beta x^{\lambda}}}$ for $x \in(0, \infty)$. Then, $p d f$ of $X$ is (2) if and only if the function $\eta$ defined in Theorem 1 has the form

$$
\eta(x)=\frac{1}{2} e^{-e^{\alpha x^{\gamma}}+e^{-\beta x^{\lambda}}}, \quad x>0 .
$$

Proof. Let $X$ have $p d f(2)$, then

$$
\{1-F(x)\} E[g(X) \mid X \geqslant x]=\frac{1}{2} e^{-2\left(e^{\alpha x^{\gamma}}-e^{-\beta x^{\lambda}}\right)}, \quad x>0,
$$

and

$$
\eta(x)-g(x)=-\frac{1}{2} e^{-e^{\alpha x^{\gamma}}+e^{-\beta x^{\lambda}}}<0 \quad \text { for } \quad x>0 .
$$

Conversely, if $\eta(x)$ is given by (3), then

$$
s^{\prime}(x)=\frac{\eta^{\prime}(x)}{\eta(x)-g(x)}=\left(\alpha \gamma x^{\gamma-1} e^{\alpha x^{\gamma}}+\beta \lambda x^{\lambda-1} e^{-\beta x^{\lambda}}\right),
$$

from which we obtain

$$
s(x)=e^{\alpha x^{\gamma}}-e^{-\beta x^{\lambda}}, \quad x>0 .
$$

Now, in view of Theorem 1, X has $c d f$ (1) and $p d f(2)$. 
Corollary 1. Let $X: \Omega \rightarrow(0, \infty)$ be a continuous random variable. Then, $p d f$ of $X$ is (2) if and only if there exist functions $g$ and $\eta$ defined in Theorem 1 satisfying the following differential equation

$$
s^{\prime}(x)=\frac{\eta^{\prime}(x)}{\eta(x)-g(x)}=\left(\alpha \gamma x^{\gamma-1} e^{\alpha x^{\gamma}}+\beta \lambda x^{\lambda-1} e^{-\beta x^{\lambda}}\right) \quad x>0 .
$$

Remarks 1. (a) The general solution of the differential equation (4) is

$$
\begin{aligned}
\eta(x)= & e^{e^{\alpha x^{\gamma}}-e^{-\beta x^{\lambda}}}\left\{-\int g(x)\left(\alpha \gamma x^{\gamma-1} e^{\alpha x^{\gamma}}+\beta \lambda x^{\lambda-1} e^{-\beta x^{\lambda}}\right)\right. \\
& \left.\times e^{-e^{\alpha x^{\gamma}}+e^{-\beta x^{\lambda}}} d x+D\right\}
\end{aligned}
$$

for $x>0$, where $D$ is a constant. One set of functions $(g, \eta)$ satisfying the above equation is given in Proposition 1 for $D=0$.

(b) Clearly there are other pairs of functions $(g, \eta)$ satisfying the conditions of Theorem 1. We presented one such pair in Proposition 1.

\section{Characterization Based on Hazard Function}

For the sake of completeness, we state the following definition.

Definition 1. Let $F$ be an absolutely continuous distribution with the corresponding $p d f f$. The hazard function corresponding to $F$ is denoted by $h_{F}$ and is defined by

$$
h_{F}(x)=\frac{f(x)}{1-F(x)}, \quad x \in \operatorname{Supp} F,
$$

where Supp $F$ is the support of $F$.

It is obvious that the hazard function of a twice differentiable distribution function satisfies the first order differential equation 


$$
\frac{h_{F}^{\prime}(x)}{h_{F}(x)}-h_{F}(x)=q(x)
$$

where $q(x)$ is an appropriate integrable function. Although this differential equation has an obvious form since

$$
\frac{h_{F}^{\prime}(x)}{h_{F}(x)}-h_{F}(x)=\frac{f^{\prime}(x)}{f(x)},
$$

for many univariate continuous distributions (5) seems to be the only differential equation in terms of the hazard function. The goal of the characterization based on hazard function is to establish a differential equation in terms of hazard function, which has as simple form as possible and is not of the trivial form (5). Here, we present a characterization of the of NMW model based on a nontrivial differential equation in terms of the hazard function.

Proposition 2. Let $X: \Omega \rightarrow(0, \infty)$ be a continuous random variable. Then, $X$ has $p d f(2)$ if and only if its hazard function $h_{F}$ satisfies the differential equation

$$
\begin{aligned}
h_{F}^{\prime}(x)+x^{-1} h_{F}(x)= & \alpha \gamma^{2} x^{\gamma-2}\left(1+\alpha x^{\gamma}\right) e^{\alpha x^{\gamma}} \\
& +\beta \lambda^{2} x^{\lambda-2}\left(1-\beta x^{\lambda}\right) e^{-\beta x^{\lambda}}, \quad x>0 .
\end{aligned}
$$

Proof. If $X$ has $p d f(2)$, then clearly (6) holds. Now, if (6) holds, then after multiplying both sides of (6) by $x$, we arrive at

$$
\frac{d}{d x}\left\{x h_{F}(x)\right\}=\frac{d}{d x}\left\{\alpha \gamma x^{\gamma} e^{\alpha x^{\gamma}}+\beta \lambda x^{\lambda} e^{-\beta x^{\lambda}}\right\},
$$

from which we have

$$
h_{F}(x)=x^{-1}\left\{\alpha \gamma x^{\gamma} e^{\alpha x^{\gamma}}+\beta \lambda x^{\lambda} e^{-\beta x^{\lambda}}\right\},
$$

or

$$
h_{F}(x)=\frac{f(x)}{1-F(x)}=\alpha \gamma x^{\gamma-1} e^{\alpha x^{\gamma}}+\beta \lambda x^{\lambda-1} e^{-\beta x^{\lambda}} .
$$

Integrating both sides of (7) from 0 to $x$, we arrive at

$$
-\ln \{1-F(x)\}=e^{\alpha x^{\gamma}}-e^{-\beta x^{\lambda}} .
$$


from which we obtain

$$
F(x)=1-e^{-e^{\alpha x^{\gamma}}+e^{-\beta x^{\lambda}}}, \quad x \geqslant 0 .
$$

\section{Characterizations Based on Single Function of the Random Variable}

In this section we employ a single function $\psi$ of $X$ and state characterization results in terms of $\psi(X)$. The following proposition has already appeared in a Technical Report (Hamedani, 2013), so we will just state it here for the sake of completeness.

Proposition 3. Let $X: \Omega \rightarrow(a, b)$ be a continuous random variable with cdf $F$. Let $\psi(x)$ be a differentiable function on $(a, b)$ with $\lim _{x \rightarrow a} \psi(x)=1$. Then for $\delta \neq 1$,

$$
E[\psi(X) \mid X>x]=\delta \psi(x), \quad x \in(a, b),
$$

if and only if

$$
\psi(x)=\{1-F(x)\}^{\frac{1}{\delta}-1}, \quad x \in(a, b) .
$$

Remark 2. Taking, e.g., $\psi(x)=e^{-\left(\frac{1-\delta}{\delta}\right)\left(e^{\alpha x^{\gamma}}-e^{-\beta x^{\lambda}}\right)}$, Proposition 3 gives a characterization of NMW distribution.

\section{Characterizations Based on Truncated Moment of Certain Functions of the 1st Order Statistic}

Let $X_{1: n} \leqslant X_{2: n} \leqslant \ldots \leqslant X_{n: n}$ be $n$ order statistics from a continuous $c d f F$. We present here a characterization result base on some functions of the $1^{\text {st }}$ order statistic. Our characterization will be a consequence of the following proposition, which is an extended version of the one appeared in our previous work (Hamedani, 2010). 
Proposition 4. Let $X: \Omega \rightarrow(a, b)$ be a continuous random variable with $c d f F$. Let $\psi(x)$ and $q(x)$ be two differentiable functions on $(a, b)$ such that $\lim _{x \rightarrow b} \psi(x)[1-F(x)]^{n}=0$ and $\int_{a}^{b} \frac{q^{\prime}(t)}{[q(t)-\psi(t)]} d t=\infty$. Then

$$
E\left\{\psi\left(X_{1: n}\right) \mid X_{1: n}>t\right\}=q(t), \quad t>a,
$$

implies

$$
F(x)=1-\exp \left[-\int_{a}^{x} \frac{q^{\prime}(t)}{n\{\psi(t)-q(t)\}} d t\right], \quad a \leqslant x<b .
$$

Remark 3. Take, e.g., $\psi(x)=e^{n\left(e^{e^{\alpha x^{\gamma}}-e^{-\beta x^{\lambda}}}\right)}, q(x)=\frac{1}{2} \psi(x)$ and $(a, b)=$ $(0, \infty)$. Clearly there are other suitable functions $\psi(x)$ and $q(x)$.

\section{References}

Doostmardi, A., Zadkarami, M.R. and Roshani Sheykhabad, A. (2014). A New Modified Weibull Distribution and Its Applications. Journal of Statistical Research of Iran, 11, 97118.

Glänzel, W. (1987). A Characterization Theorem Based on Truncated Moments and Its Application to Some Distribution Families, Mathematical Statistics and Probability Theory (Bad Tatzmannsdorf, 1986), Vol. B, Reidel, Dordrecht, 75-84.

Hamedani, G.G. (2010). Characterizations of Continuous Univariate Distributions Based on the Truncated Moments of Functions of Order Statistics, Studia Sci. Math. Hungar., 47, 462-484.

Hamedani, G.G. (2013). On Certain Generalized Gamma Convolution Distributions II, Technical Report, No. 484, MSCS, Marquette University.

\section{G. G. Hamedani}

Department of Mathematics, Statistics

and Computer Science,

Marquette University,

Milwaukee, WI 53201-1881 USA.

email: gholamhoss.hamedani@marquette.edu 